\title{
ASSESSING THE EFFECT OF DIFFERENTIAL DIFFUSION FOR STRATIFIED LEAN PREMIXED TURBULENT FLAMES WITH THE USE OF LES-PDF FRAMEWORK
}

\author{
W.P. Jones, A.J.Marquis, \\ Department of Mechanical Engineering, \\ Imperial College London, London \\ K. Vogiatzaki * \\ Advanced Engineering Centre, University of Brighton, \\ Lewes Road, Brighton, UK
}

\begin{abstract}
Lean premixed stratified combustion mode is rapidly growing in importance for modern engine designs. This paper presents Large Eddy Simulations for a new burner design in order to assess the predictive capability of the probability density function (pdf) approach to flames that propagate through non homogeneous mixtures in terms of equivalence ratio. Although various efforts have been made in the past for the simulation of the same test case the novelty of this work lies to the fact that it is the first simulation effort that differential diffusion is accounted for given the relatively low Reynolds numbers $(13,800)$ of the configuration. First mean and root mean square velocity simulations are performed for the isothermal cases in order to asses the effect of the grid resolution and the overall LES flow field solver. Then instantaneous snapshots of the flame are presented in order to provide insight to the structure of the flame and the effect of stratification. Finally results for velocities, temperature and mixture fraction are presented
\end{abstract}

*corresponding author, email: K.Vogiatzaki@brighton.ac.uk 
and compared with the experimental data. Overall the results are in very good agreement with experiments.

\section{Introduction}

Pollutant formation in modern engines has been associated to the high peak temperature at stoichiometric conditions. In order to tackle this issue lean premixed combustion mode has been suggested as a potential solution and nowadays is of increasing importance in many industrial applications (including aero-engines, gas turbines for power plants and internal combustion engines). Within these devices, mixture stratification is commonly observed. While the global equivalence ratio is lean, locally significant variations in the reactants occur. This is different to idealised premixed or non-premixed conditions reproduced in well-know jet flames, where, prior to chemical reactions, reactants are either perfectly mixed on a molecular level or completely segregated. stratified lean combustion one of the most interesting areas of research for modern combustion systems which despite its significance remains vaguely understood due to the complexity of parameters that control these flows

Although a detailed review on stratified combustion can be found in the recent work of Lipatnikov [1] here we provide a short overview of existent experimental and numerical studies the findings of which will help us explain the results presented in this paper. A main difficulty in understanding stratified combustion characteristics is the rather limited number of available experimental data at well-designed generic flame configurations that could be used for numerical validation.Experimental studies of the recent years include the One Rig for Ac- 
curate Comparisons (ORACLES) rig [2], the stratified burner of Cambridge University [3, 4, 5, 6, 7, 8] and the stratified burner of Darmstadt University [9, 10]

The ORACLE experiment is one of the first experiments that was specifically designed in order to test turbulent combustion models that are able to take into account a variable-mixture composition and the simultaneous presence of largescale coherent structures and stochastic turbulence. The study concerns cases for which both streams have the same mass flow rate and the same equivalence ratio. One important finding of this study is that inert and instantenious flow patterns past the present two-stream sudden expansion are characterised by asymmetry with respect to the combusiton chamber centreline. Unlike the case of an inert wake behind a bluff body, the inert flow in the case under investigation does not show any distinguishable frequency on the velocity spectra. The most dominant effect of the presence of combusiton on the large-scale motion of the flow is to bring a quite perfect symmetry to the mean and to some extent to the unsteady flow patterns behind the sudden expansion. In this case there are marked frequencies on the velocity spectra which shows the presence of a periodic component in the large scale unsteady motion that is clearly related to the flame brush dynamics. Also a stochastic behaviour of the flame fronts exists at the smaller scales.

In some additional experimental work (also prior to 2010) stratified flame characteristics such as flame curvature, flame propagation speed and flame thickness were investigated [11, 12, 13]. It was shown that stratification can be associated with an increase to the flame propagation rate when unsteady flame kernels are of interest. This local variation in burning velocity results in increased flame front wrinkling relative to premixed flames, with a corresponding broadening of 
curvature distributions. It was also found that even in cases that the same mean equivalence ratio ahead of the flame is present following ignition in premixed and stratified cases, in reality the mean propagation speed in stratified flames was higher than in corresponding premixed cases. This enhancement is the result of the diffusion of heat and radicals from richer products into leaner reactants (ie, back-supported flame propagation). The above studies have also revealed that two families of stratification scales exist [12]: The one is the "large-scale stratification" and it is associated with a flame that propagates through a low gradient of stratification. The second is the "small-scale stratification" where the flame front passes through locally rich or lean pockets. Although in laboratory flames these two families can be separated, in practical devices, they co-exist.

At the Cambridge experiment premixed and stratified V-flames and low turbulent intensity were investigated. The results of the experiments are reported in [5, 6, 4]. The degree of stratification and swirl are systematically varied to generate a matrix of experimental conditions, allowing their separate and combined effects to be investigated. Non-swirling flows are considered in [5], and the effects of swirl are considered in a companion paper [6]. The burner is unconfined and the flame is stabilised at the central bluff body.The flames surveyed fall within the thin reaction zone regime on the modified Borghi diagram. An important issue discussed in [4] relevant to these flames is preferential species diffusion and differential diffusion of heat and mass. Preferential diffusion has important effects on local flame structure in turbulent premixed flames while differential diffusion of heat and mass can affect both local flame structure and global flame parameters, such as turbulent flame speed. This is an important characteristic of these flames that should be reflected on the models. Also results suggest that the 
large scale effects observed in the turbulent bluff-body burner are caused by preferential transport of $\mathrm{H}_{2}$ and $\mathrm{H}_{2} \mathrm{O}$ through the preheat zone ahead of $\mathrm{CO}_{2}$ and $\mathrm{CO}$, followed by convective transport downstream and away from the local flame brush. This preferential transport effect increases with increasing velocity of reactants past the bluff body and is apparently amplified by the presence of a strong recirculation zone where excess $\mathrm{CO}_{2}$ is accumulated.

The work of Haworth et al. [14] and Jimenez et al. [15] were among the first efforts to give numerical insight to stratified combustion under global lean conditions close to those in an automotive gasoline direct-injection (GDI) engine with the use of 2D Direct Numerical Simulations (DNS) of stratified propane mixtures. The focus was the investigation of the propagation speed and the heat release rate of flames in non-homogeneous premixed conditions. The studies revealed that the heat release rate is significantly increased by the distribution of mixture fraction at fuel-lean conditions. In addition in [15] two combustion models that assume that the structure of the flame can be determined from the mixture fraction were assessed. The flamelet model was proved adequate for the primary reaction zone where most of the fuel is consumed. However, for the secondary reaction zone that results from the combustion of the products of the primary zone the flamelet assumption is not anymore satisfying and a more advanced combustion model as the conditional moment closure (CMC) [16] approach seems more suitable. In the Large Eddy Simulation (LES) context most of the studies have focused either on premixed or non premixed configurations. Stratification is treated as a category of premixed configurations where the incoming mass flow rate oscillates due to flow and combustion instabilities (see for example [2]) or results due to externally forced pulsating fuel flow rates [17]. Thus most of the combustion models tested 


\begin{tabular}{|c|c|c|}
\hline Study & Flames & Model \\
\hline Roux et al [22] & $A_{r}, G_{r}$ & G-Eq. \\
\hline Kuenne et al [23] & $A_{r}$ & TF/FGM \\
\hline Marincola et al [24] & $A_{r}, G_{r}$ & FSD \\
\hline Trisjono et al [25] & $A_{r}, C_{r}$ & LS /Progress var. \\
\hline
\end{tabular}

Table 1: Previous LES studies of Darmstadt Stratified Burner . FSD stands for Flame Surface Density, LS for Level Set and TF for Thickened Flame Model.

are the traditional models for premixed flames such as the artificially thickened flame model [17] or the ones based on the flamelet concept [18, 19, 20, 21].

A stratified burner has been developed at the University of Darmstadt in order to understand the behaviour of stratified turbulent flames under free flame propagation [9, 10]. A series of 11 methane flames under different levels of stratification and with sufficiently high shear rate has been measured. One of the first modelling efforts of these flames can be found in the report of Roux and Pitch [22] where the G-equation model coupled with a transported progress variable has been used. In Table 11 a summary of the previous studies and the combustion model used is provided.

The above studies reveal some important characteristics of the combustion dynamics of the flames under investigation. Close to the pilot, combustion predominantly takes place in a homogeneous mixture, whereas further downstream combustion occurs at leaner conditions and under a wider distribution of mixture compositions [23]. Moreover, the front-supported flame configuration, occurring when a flame front propagates towards a richer mixture, is only found in the near nozzle region. In contrast, back-supported flames, characterised by a flame front propagating towards a leaner mixture, are observed at all downstream positions [23, 24]. Finally inclusion of heat loses is important for the accurate predictions 
close to the nozzle. Radiation, on the other hand does not play a role close to the nozzle however is important further downstream [25].

The current paper aims at investigating numerically the structure one of these new flames. The main objective is to asses the performance of the Probability Density Function ( $p d f)$ approach( [26]) -traditionally known as an accurate model for non-premixed combustion- for the prediction of the stratification effects. LES have been used for the solution of the flow field equations and the Eulerian Stochastic field approach for the solution of the one-point $p d f$ of the joint composition has been chosen in order to descried the turbulence chemistry interactions. Following the approach of Brauner et al [27] accurate transport properties are incorporated so that differential diffusion are included. The paper is organised as follows. Section 2 describes the model and the differential diffusion modelling. Section 3 describes the experimental configuration and information are provided for the numerical calculations. Results for both isothermal and reactive test cases are presented in section 4 and then follows the conclusions.

\section{Mathematical Formulation}

In the present work a filter with a top-hat shape, i.e. implicit with the filter width $\Delta$ related to the local cube root of the grid volume, is used to obtain the filtered transported equations. Together with Favre filtering, to account for the variation of density as a consequence of combustion, the continuity and Navier-Stokes equations become:

$$
\frac{\partial \bar{\rho}}{\partial t}+\frac{\partial \bar{\rho} \tilde{u}_{i}}{\partial x_{i}}=0
$$




$$
\frac{\partial \bar{\rho} \tilde{u}_{i}}{\partial t}+\frac{\partial \bar{\rho} \tilde{u}_{i} \tilde{u}_{j}}{\partial x_{j}}=-\frac{\partial \bar{p}}{\partial x_{i}}-\frac{\partial \tau_{i j}}{\partial x_{j}}+\frac{\partial}{\partial x_{j}} \bar{\mu}\left(\frac{\partial \tilde{u}_{i}}{\partial x_{j}}+\frac{\partial \tilde{u}_{j}}{\partial x_{i}}-\frac{2}{3} \frac{\partial \tilde{u}_{k}}{\partial x_{k}} \delta_{i j}\right)
$$

where the symbols represent their usual quantities. The sub-grid scale $(s g s)$ stress, defined as $\tau_{i j}=-\bar{\rho}\left(\widetilde{u_{i} u_{j}}-\tilde{u}_{i} \tilde{u}_{j}\right)$ is closed using a dynamic version of the Smagorinsky model [28]:

$$
\mu_{s g s}=\bar{\rho}\left(C_{S} \Delta\right)^{2}\left\|\tilde{e}_{i j}\right\|
$$

where $\left\|\tilde{e}_{i j}\right\| \equiv \sqrt{2 \tilde{e}_{i j} \tilde{e}_{i j}}$ is the Frobenius norm of the resolved rate of strain tensor, $\tilde{e}_{i j}=0.5\left(\frac{\partial \tilde{u}_{i}}{\partial x_{j}}+\frac{\partial \tilde{u}_{j}}{\partial x_{i}}\right)$. The filter width is taken as the cube root of the local grid cell volume and the parameter $C_{s}$ is obtained through the dynamic procedure of Piomelli and Liu [29]. As with the viscous stress the isotropic part of the sgs stress is adsorbed into the pressure. The transport equations for enthalpy and specific mole number $[\mathrm{kmol} / \mathrm{kg}]$ of the chemical species can be expressed as:

$$
\begin{gathered}
\frac{\partial \bar{\rho} \tilde{h}}{\partial t}+\frac{\partial \bar{\rho} \tilde{u}_{j} \tilde{h}}{\partial x_{j}}=-\frac{\partial \bar{J}_{h, j}}{\partial x_{j}}-\frac{\partial\left(\overline{\rho u_{j} h}-\bar{\rho} \tilde{u}_{j} \tilde{h}\right)}{\partial x_{j}} \\
\frac{\partial \bar{\rho} \tilde{n}_{\alpha}}{\partial t}+\frac{\partial \bar{\rho} \tilde{u}_{j} \tilde{n}_{\alpha}}{\partial x_{j}}=-\frac{\partial \bar{J}_{\alpha, j}}{\partial x_{j}}+\overline{\rho \dot{\omega}_{\alpha}(\mathbf{n}, \mathbf{T})}-\frac{\partial\left(\overline{\rho u_{j} n_{\alpha}}-\bar{\rho} \tilde{u}_{j} \tilde{n}_{\alpha}\right)}{\partial x_{j}}
\end{gathered}
$$

where the heat flux is given by:

$$
J_{h, j}=-\frac{\mu}{\sigma} \frac{\partial h}{\partial x_{j}}+\sum_{\alpha=1}^{N_{s}} h_{\alpha}\left(J_{\alpha}+\frac{\mu}{\sigma} \frac{\partial n_{\alpha}}{\partial x_{j}}\right)
$$


where $\sigma$ is the Prandtl number. and where the species diffusive flux is given by, [30]:

$$
J_{\alpha, j}=-\rho D_{\alpha}\left(\frac{\partial n_{\alpha}}{\partial x_{j}}-\frac{n_{\alpha}}{n} \frac{\partial n}{\partial x_{j}}\right)
$$

where $h_{\alpha}$ is enthalpy of species $\alpha$ and $D_{\alpha}$ the diffusivity of species $\alpha$ and where $n$ is the specific mole number of the mixture. The diffusive fluxes given by equation (6) do not necessarily sum to zero and a correction velocity, [31], independent of species, is therefore added to ensure mass conservation. In the present work sub-grid variations in the transport properties, i.e. viscosity, Prandtl number etc, are neglected.

The main difficulty in LES of combustion processes is the closure of the filtered chemical source term appearing in Equation (4). For this it is possible to use a one-point joint filtered $p d f$ for all the scalar quantities required to describe the reaction. The transport equation of such a $p d f$ provides a means of describing the temporal and spatial variation of the scalars one-point statistics and has the advantage that the chemical source term appears in closed form.

The density-weighted filtered joint pdf for a set of scalars $\psi$ needed to describe a reaction can be defined as [32]

$$
P_{s g s}(\psi ; x, t)=\int_{\Omega} \prod_{\alpha=1}^{N s} \delta\left(\psi_{\alpha}-\phi_{\alpha}(\mathbf{x}, \mathbf{t})\right) \mathbf{G}\left(\mathbf{x}-\mathbf{x}^{\prime} ; \boldsymbol{\Delta}\right) d \mathbf{x}^{\prime}
$$

where $\delta$ is a Dirac delta function.

This pdf can be used to describe the behaviour of scalars on the sub-filter level by essenti]ally describing the probability of $\phi=\psi$ arising inside a filter volume, thus providing the information necessary to evaluate the filtered chemical source 
term. Following the approach of [33, 27] the resolved part of the sub-grid 'mixing' term is subtracted from both sides of the equation and so that the resulting pdf evolution equation can be written:

$$
\begin{aligned}
\frac{\partial \bar{\rho} \tilde{P}(\underline{\psi})}{\partial t} & +\frac{\partial \bar{\rho} \tilde{u}_{j} \tilde{P}(\underline{\psi})}{\partial x_{j}}-\sum_{\alpha=1}^{N} \frac{\partial J_{\alpha, i}(\underline{\bar{\phi}})}{\partial x_{i}} \frac{\partial \bar{P}(\underline{\psi})}{\partial \psi_{\alpha}}+\sum_{\alpha=1}^{N} \frac{\partial}{\partial \psi_{\alpha}} \bar{\rho} \dot{\omega}_{\alpha}(\underline{\psi}) \tilde{P}(\underline{\psi})+ \\
& =-\frac{\partial\left(\overline{\rho u_{j} \mathcal{F}(\underline{\psi})}-\bar{\rho} \tilde{u}_{j} \tilde{P}(\underline{\psi})\right)}{\partial x_{j}} \\
& +\sum_{\alpha=1}^{N} \frac{\partial}{\partial \psi_{\alpha}}\left(\left[E\left(\frac{\partial J_{\alpha, i}(\underline{\phi})}{\partial x_{i}}-\frac{\partial J_{\alpha, i}(\underline{\phi})}{\partial x_{i}}\right) \mid(\underline{\psi})\right] \bar{P}(\underline{\psi})\right)
\end{aligned}
$$

where . and - represent density weighted and unweighed filtered quantities and where $J_{\alpha, i}$ is the diffusive transport of the scalar $\phi_{\alpha}$ - equation (5) or (6) as appropriate. In this formulation the chemical source term does not require closure; however, the last three terms on the r.h.s. do. The first, the sub-grid transport of the pdf, can be closed using a gradient closure similar to the Smagorinsky model. The last two terms, representing the effect of molecular diffusion on the pdf, cannot be represented due to the pdfs one-point nature [26]. It is therefore modelled by introducing a micro-mixing term that represents the sub-grid diffusion, which is closed using a linear mean square estimation approach [34]. Thus the two modelled terms on the rhs. can be written as:

$$
\begin{gathered}
-\frac{\partial}{\partial x_{j}}\left[\left(\frac{\mu_{s g s}}{\sigma_{s g s}}\right) \frac{\partial \tilde{P}_{s g s}(\underline{\psi})}{\partial x_{j}}\right] \\
C_{d} \frac{\bar{\rho}}{\tau_{s g s}} \sum_{\alpha=1}^{N_{s}} \frac{\partial}{\partial \psi_{\alpha}}\left[\left(\psi_{\alpha}-\phi_{\alpha}(\underline{x}, t)\right) \tilde{P}_{s g s}(\underline{\psi})\right]
\end{gathered}
$$


The sgs-mixing time scale $\tau_{s g s}$ is defined as $\frac{\bar{\rho} \Delta^{2}}{\mu_{s g s}}$. For well resolved and low turbulence areas this has the desired effect of reducing the pdf to a $\delta$-function.

The approach used to handle the high dimensionality of the pdf method is the Eularian stochastic field method, where the transport of the pdf is represented by a system of stochastic differential equations that is equivalent to the modelled transport equation [35], [36]. The Ito formulation of the stochastic integral is used and the equation governing the evolution of the fields can be written as:

$$
\begin{aligned}
\bar{\rho} d \xi_{\alpha}^{n}=- & \bar{\rho} \tilde{u}_{i} \frac{\partial \xi_{\alpha}^{n}}{\partial x_{i}} d t-\frac{\partial J_{i, \alpha}}{\partial x_{i}} d t+\frac{\partial}{\partial x_{i}}\left[\frac{\mu_{s g s}}{\sigma_{s g s}} \frac{\partial \xi_{\alpha}^{n}}{\partial x_{i}}\right] d t \\
& +\left(2 \bar{\rho} \frac{\mu_{s g s}}{\sigma_{s g s}}\right)^{1 / 2} \frac{\partial \xi_{\alpha}^{n}}{\partial x_{i}} d W_{i}^{n}-\frac{C_{d}}{2} \frac{\bar{\rho}}{\tau_{s g s}}\left(\xi_{\alpha}^{n}-\tilde{\phi}_{\alpha}\right) d t+\bar{\rho} \dot{\omega}_{\alpha}^{n}\left(\xi^{n}\right) d t
\end{aligned}
$$

where $J_{i, \alpha}$ is given by equation $(5)$ or $(6)$ as appropriate. $\tilde{P}_{s g s}$ is represented by an ensemble of $N_{s}$ stochastic fields for each of the $N$ species included in the reaction mechanism. This stochastic approach has the benefit that computational cost increases linearly with complexity; a more detailed discussion of the method can be found in e.g. [37].

\section{Computational methods}

Experiments were performed in lean flames stabilised by a central premixed pilot. The burner consists of three concentric tubes with inner diameter of $D_{p}=14.8$, $D_{s_{1}}=37 \mathrm{~mm}$ and $D_{s_{2}}=60 \mathrm{~mm}$. The tubes are staged in order to enable full optical access to the exit profiles (see Fig. 11). The burner is placed inside a $600 \mathrm{~mm}$ wide air-co-flow with velocity of $0.1 \mathrm{~m} / \mathrm{s}$. 


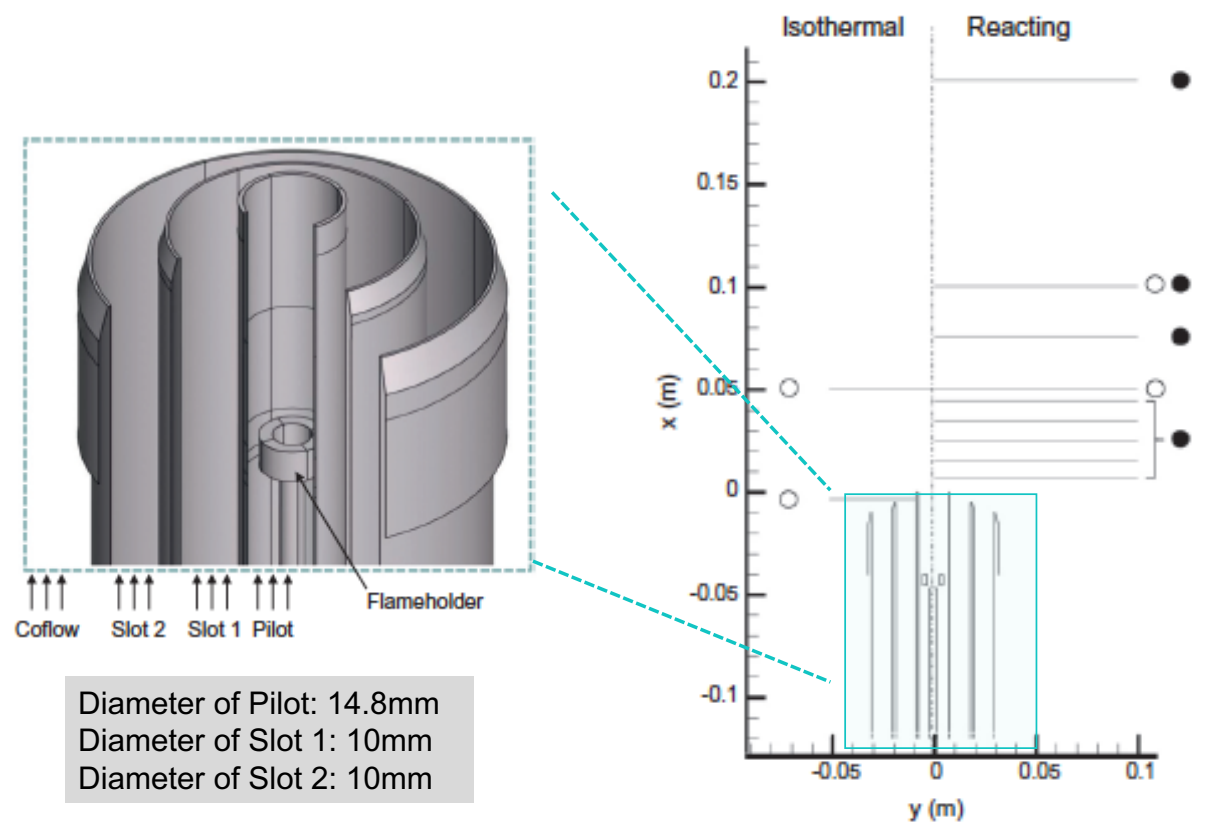

Figure 1: Pipe geometry (left) along with a cut at the exit region (right). Points indicate the location where experimental data were collected [23]

LES calculations were performed for a reactive case of the operating conditions at which DLR-AT (TSFA) performed measurements. The characteristic of this case is that there is minimum shear and stratification between the pilot and the first slot and stratification between slots 1 and 2. Additional isothermal simulations were performed in order to assess weather the grid used for the reactive cases offers a reasonable resolution. The isothermal case is indicated by the index 'i2' and the reacting simulations indicated by the index ' $r$ '. The simulations are performed with filter width equal to the cube root of the local grid cell volume and a dynamic version of the Smagorinsky model [28] is used for the sgs stresses. The deviatoric part of the sub-grid stress is related to the filtered rate of strain tensor via $\tau_{i j}^{s g s}=\mu_{s g s} \widetilde{S}_{i j}$ with the $s g s$ viscosity given by $\mu_{s g s}=\bar{\rho}\left(C_{s} \Delta^{2}\right)\left\|\widetilde{S}_{i j}\right\| .\left\|\widetilde{S}_{i j}\right\|$ represents a Frobenius norm. 
The flow conditions are demonstrated in Table 2. Two different grids are used for the simulation of these flow configurations (see Table 3): one coarse grid of the order of 1 million cells and a second much finer grid of the order of 6 million cells. The Kolmogorov length scale based on the isothermal cases Re numbers and the diameter of the pilot is $0.01 \mathrm{~mm}$ for TSFA. Consequently even the fine grid for this case is one order of magnitude greater than the smallest flow scale. Fully developed turbulence at the exit of the slots 1 and 2 is ensured at the experiments by radial drill holes of $5 \mathrm{~mm}$ diameter at the tubes inlets. In our simulations the tubes are excluded from the computational domain and the inlet conditions are imposed from the measurements at $1 \mathrm{~mm}$ downstream for the isothermal case $T S F A_{i 2}$. Thus, the turbulence is generated in the calculations due to shear and not by the use of an artificial turbulence geenrator. For the reactive case $T S F A_{r}$, the inlet condition were imposed by experimental data that were available at $1 \mathrm{~mm}$ downstream for intermediate conditions where the pilot is burning but the other two streams are filled with air (indicated as TSFAi2 in [9. [10]). It is worth mentioning that in none of the previous studies of these flame the full pipe configuration is included in the calculations this would increase the computational cost. Only at the study of [23] close to realistic inlet conditions are simulated. The computational domain extends at $120 \mathrm{~mm}$ (out of $500 \mathrm{~mm}$ ) inside the pipes and the perforated plate inside the pilot is also included. To allow though the turbulent structures to form on this reduced length the measured profiles of the time averages axial locations at $\mathrm{x}=-4$ and $\mathrm{x}=-9 \mathrm{~mm}$ for the inner and outer slot respectively were set at the inlet. Furthermore artificial turbulence producing spatial and temporal correlated velocity fluctuations were superimposed. In this way although the geometry is realistic the actual turbulence generation method is 


\begin{tabular}{|c|c|c|c|c|c|c|c|}
\hline $\mathrm{TSF}$ & $\phi_{\text {pilot }}$ & $u_{\text {pilot }}$ & $\phi_{\text {slot } 1}$ & $u_{s 1}$ & $\phi_{\text {slot } 2}$ & $u_{s 2}$ & Re $_{\text {slot } 2}$ \\
\hline $\mathrm{Ai} 2$ & 0 & 10 & 0 & 10 & 0 & 10 & \\
\hline $\mathrm{Ar}$ & 0.9 & 1 & 0.9 & 10 & 0.6 & 10 & 13,300 \\
\hline
\end{tabular}

Table 2: Operating conditions for the simulated cases. TSF stands for "turbulent stratified flames". The isothermal case is indicated by the index ' 12 ' and the reacting simulations indicated by the index ' $r$ '.

\begin{tabular}{|c|c|c|}
\hline Grid & Number of cells & Smallest resolution \\
\hline Grid1 $(\mathrm{G} 1)$ & $1 \times 10^{6}$ & $3 \mathrm{~mm}$ \\
\hline Grid2 (G2) & $6 \times 10^{6}$ & $1.5 \mathrm{~mm}$ \\
\hline
\end{tabular}

Table 3: Grid parameters

artificial.

The in-house block-structured, parallel, boundary conforming coordinate LES code, BOFFIN-LES has been used for the calculations presented in this paper. The code is based on a finite-volume approach using an implicit low-Mach number formulation with an approximate factorisation method for the pressure. A summary of the different CFD calculations performed is given in Table 3 .

\section{Results and discussion}

\subsection{Isothermal Case TSFAi2}

Results for the isothermal case $T S F A_{i 2}$ are presented in this section. The sensitivity to the grid resolution is assessed. Calculations are performed with two different grids. Details regarding the grids can be found in table 2. Time averaged velocity statistics are shown in Figs 2 and 3 for two axial locations. The three 'bumps' at the mean axial velocity profiles at $\mathrm{z}=50 \mathrm{~mm}$ are due to the wall bounded flow at the inlet caused by the tripartite gas feed however further down- 

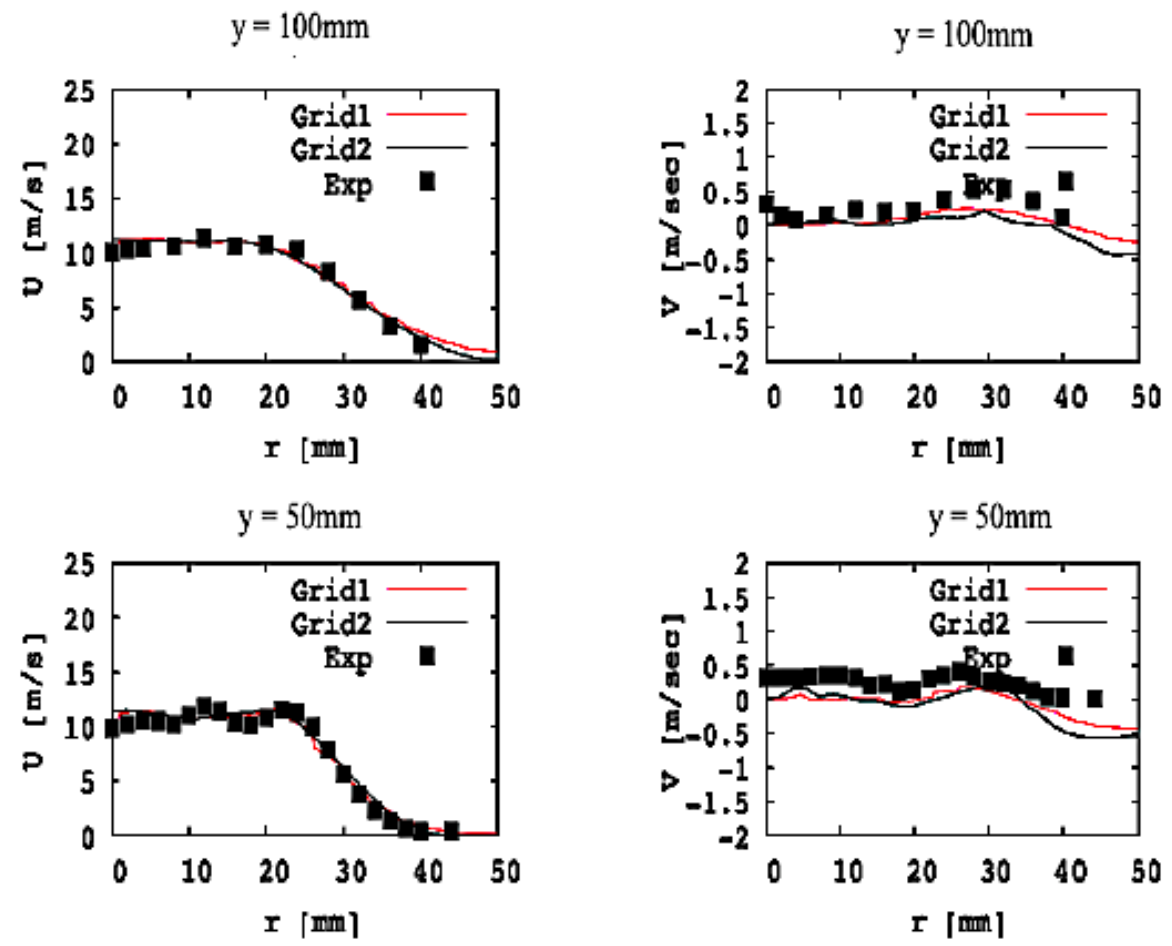

Figure 2: Radial profiles of axial (left) and radial (right) velocity for TSFAi2 at two axial locations

stream these minima tends to disappear. In addition the mean axial velocity is positive everywhere which implies there is no recirculation zone downstream of the tube exit behaviour that is also reproduced in the simulations. In the calculation these trends are accurately reproduced. Mean axial velocity is predicted very accurately and the differences between the two grids are very small. Some small discrepancies on the grid are noticed at the prediction of the radial velocity which are attributed to the omission at the simulations of the flow in the tubes. The peak of RMS around $r=30 \mathrm{~mm}$ is due to the shear between slot 2 and the co-flow. This peak is captured much better when a finer grid is used. Overall the predictions are very good even when a coarse grid is used and the qualitative as well as quantita- 

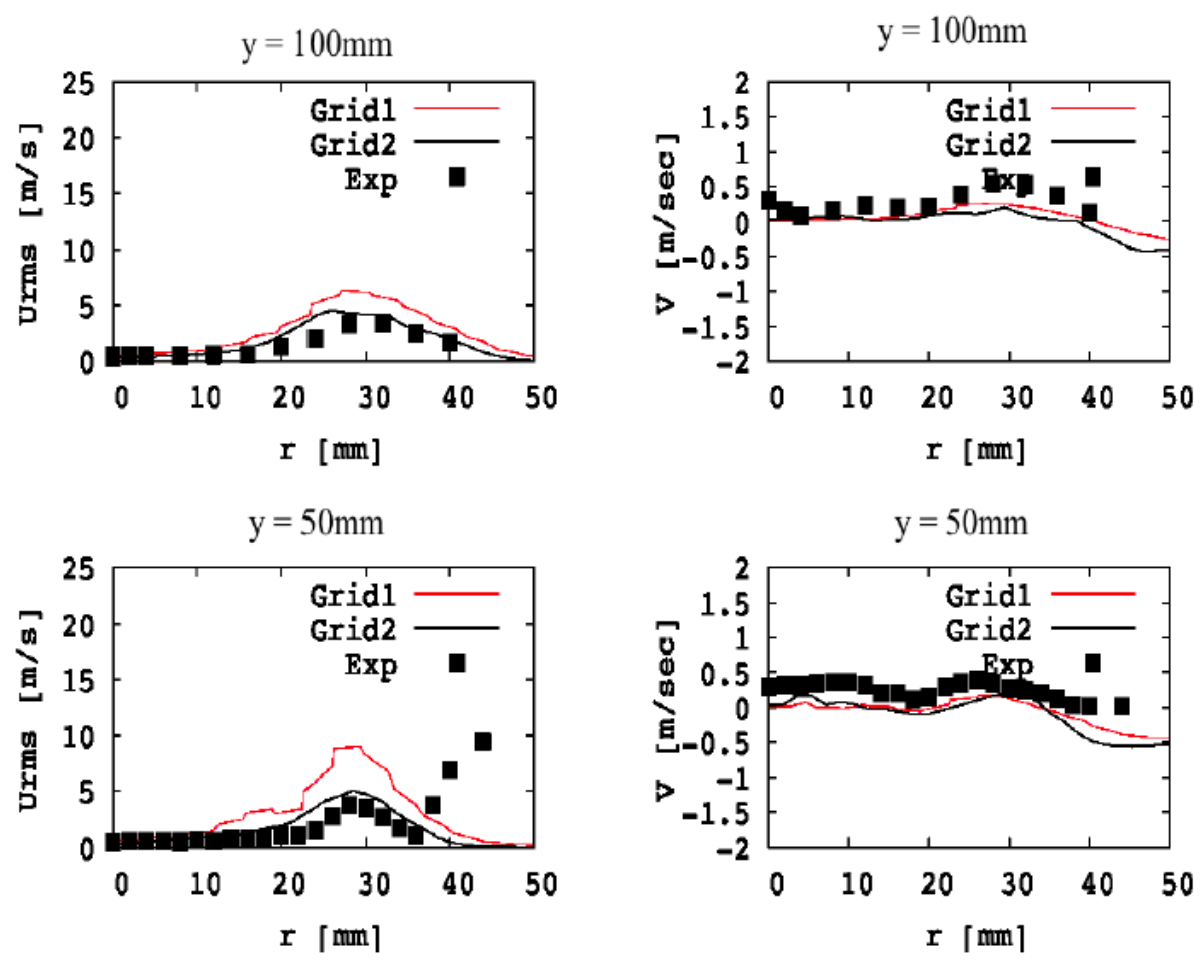

Figure 3: Radial profiles of axial and radial velocity rms for TSFAi2 at two axial locations

tive characteristics of the flames are captured. It should be noticed that the finer grid (grid 2) requires almost three times the computational time of the coarser grid (grid 1).

\subsection{Reactive Case TSF $A_{r}$}

Following the validation of the approach for the non-reactive case we extend our study in this section to the reactive case TSFAr. Starting with a qualitative analysis the instantaneous flame dynamics are presented. Simulations with and without differential diffusion are included. Figure 4, 5 and 6 provide insight into the flame structure as well as the effect of stratification on combustion process. The 

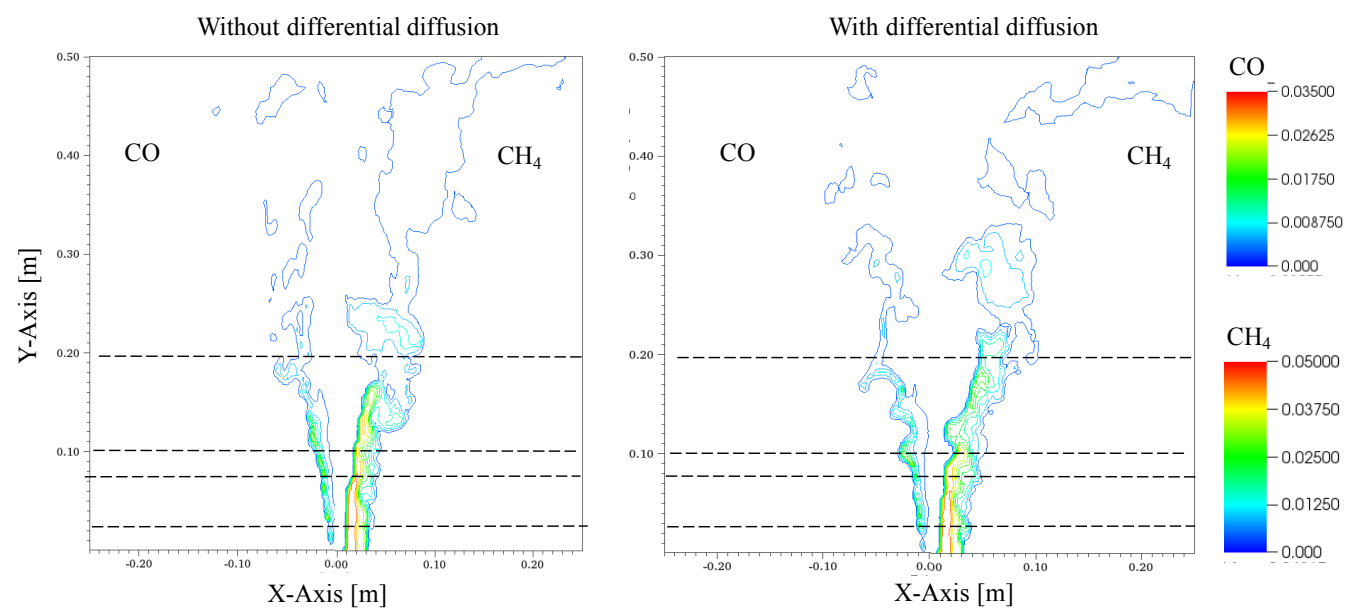

Figure 4: Instantaneous Iso-contours of $\mathrm{CH}_{4}$ (left) and $\mathrm{CO}$ (right) for simulations performed with (right) and without (left) differential diffusion accounted for

dashed lines in Figs. 4, 5 indicate the locations where the slices of Fig. 6 were taken. Hot exhaust gases of a mixture of methane with equivalent ratio of 0.9 exit from the pilot tube (see the light orange circle at $\mathrm{Y}=25 \mathrm{~mm}$ at Fig. 6). As we move further downstream these gases ignite the fresh gases of slot 1 and mixing is intensified between the fuel of slot 2 and the co-flow. In Fig. $4 \mathrm{CH}_{4}$ iso-surfaces can be considered as an indicator of the mixing process among the slots, while $\mathrm{CO}$ is a flame brush indicator. It can be seen that the flame brush for both calculations (with and without differential diffusion) is relatively flat although for the case of differential diffusion some more wrinkles are present from $\mathrm{Y}=25 \mathrm{~mm}$ up to $200 \mathrm{~mm}$. The Reynolds number of this flame and burning is likely to result in laminarisation in parts of the flow. This is born out by the values of $s g s$ viscosity which are very small over significant parts of the flame. In these circumstances molecular diffusion is likely to play a dominant role. This is confirmed by the fact that $\mathrm{CH}_{4}$ iso-contours appear to be more diffused when differential diffusion is included. It is also interesting to notice that through the intantenious plots of $\mathrm{H}_{2}$ 

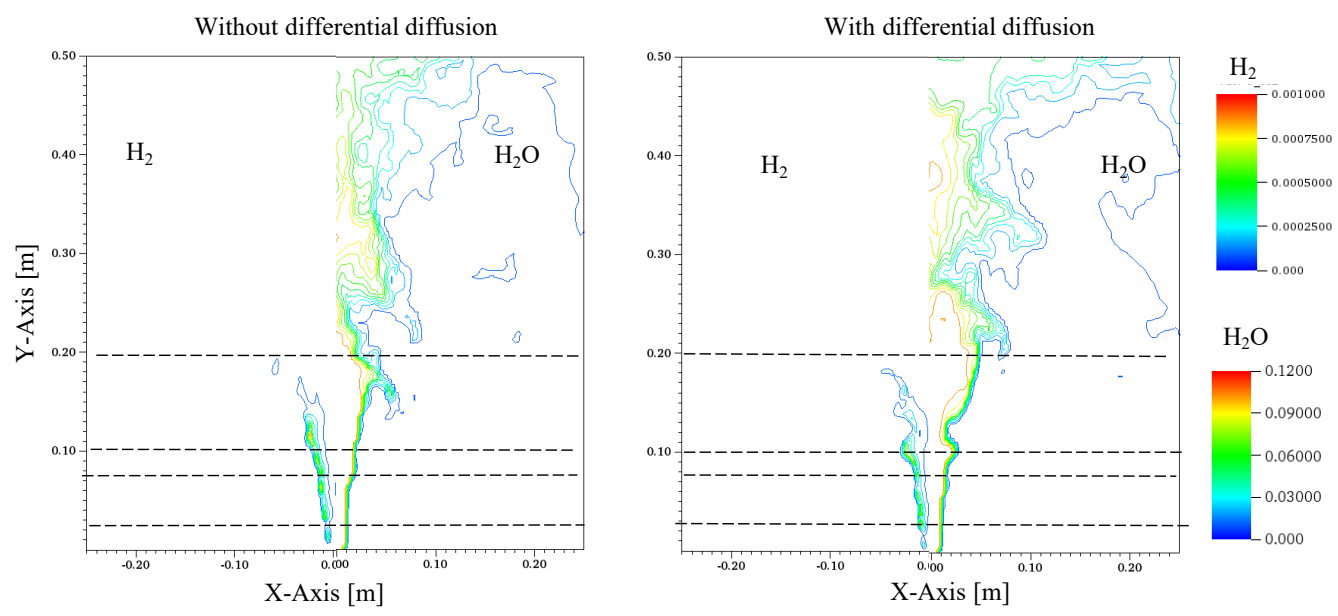

Figure 5: Instantaneous Iso-contours of $\mathrm{H}_{2}$ (left) and $\mathrm{H}_{2} \mathrm{O}$ (right) for simulations performed with (right) and without (left) differential diffusion accounted for

and $\mathrm{H}_{2} \mathrm{O}$ higher wrinkiling in their respoctive iso-surfaces is seen when differential diffusion is included .This is consistent with the findings of the study in [4]. In this study it was reported that $\mathrm{H}_{2}$ and $\mathrm{H}_{2} \mathrm{O}$ diffuse preferentially ahead of $\mathrm{CO}_{2}$ and $C O$ toward the reactants and are subsequently transported downstream and away from the local flame brush. It should though be pointed out that in the study of Barlow et al the experimental configuraiton is a turbulent bluff-body-stabilized flame and thus the recirculation zone affect to a large extent these observations. Looking at Fig. 6 we can see that the elevated temperature on flame centre initially is causes by the hot pilot gases but then is the result of intermittent flame cusps burning upstream (see Fig. 4). Although in the first three locations the temperature contours appear to be almost identical with and without differential diffusion further downstream the flame behaviour is different. Less burning is occurring in the centre (white area) while the flame front is much more wrinkled. Increased heat release is known to suppress local flow vortices since viscosity is increased. By accounting for differential diffusion this effect is better captured. 
Without differential diffusion
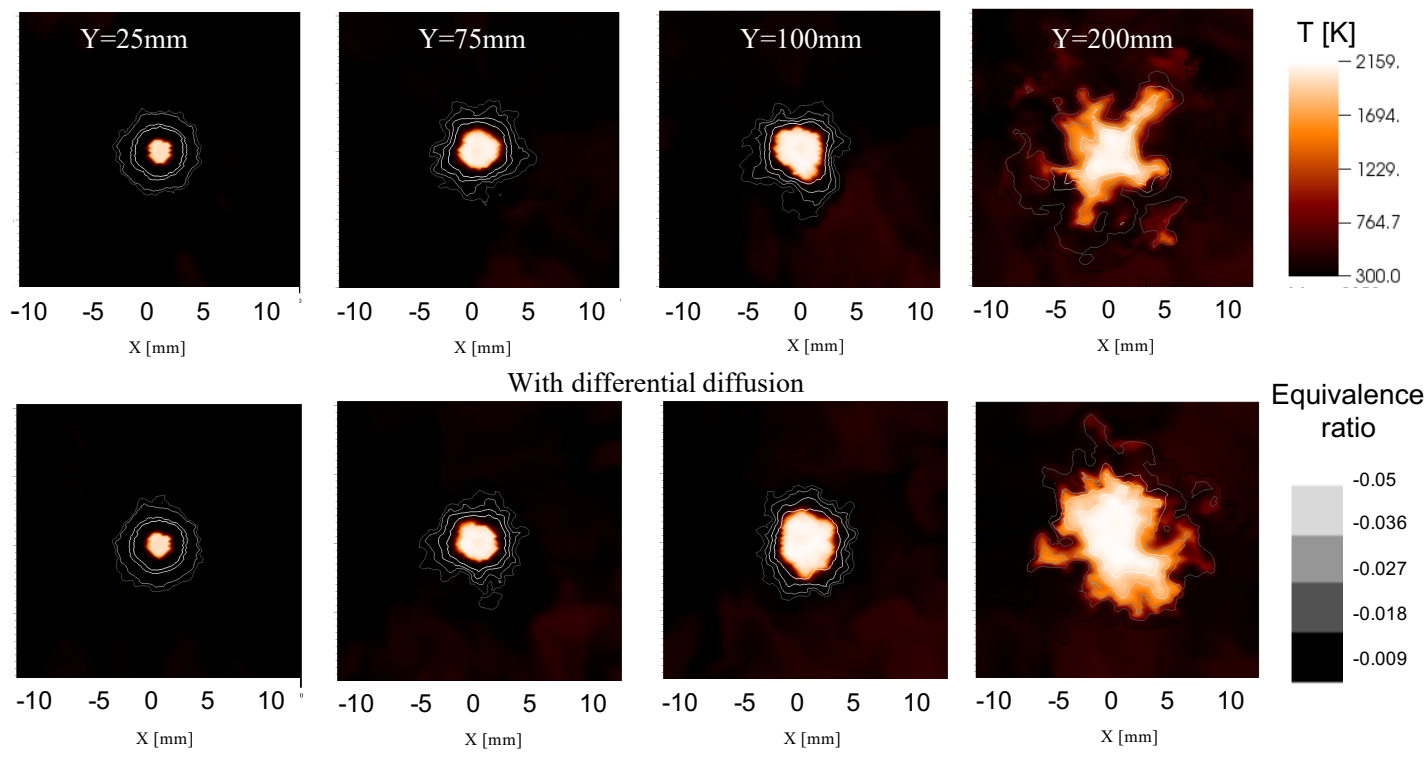

Figure 6: Slices of Instantaneous contours of temperature (orange scale) and isocontours of equivalence ratio (gray scale). The black square indicates a frame of 20x20mm.

Figures 7 and 8 show the comparison of the radial profiles of axial velocity, mixture fraction, temperature and temperature rms at various radial locations. Experimental data are available for $Y>25 \mathrm{~mm}$ The simulated profiles are in good overall agreement with the experiment data and very small differences are noticed when the effect of differential diffusion is accounted for. The simulated profiles of all four quantities are in almost perfect agreement with the measurements at a downstream distance of $50 \mathrm{~mm}$ and $200 \mathrm{~mm}$ although discrepancies are noticed at the intermediate locations. The most challenging comparison is the temperature rms. Both the comparison for the temperature and temperature rms indicate that the flame opening is not captured accurately. However this might be a result of the fact that the pipe flow and radiation effects are not included, rather than a sign that the effect of differential diffusion is small. 


\section{Conclusion}

In this work the $p d f$ approach was used to perform LES of a lean stratified burner studied experimentally at the university of Darmstadt. The approach is based on the transformation of the joint species $p d f$ transport equation stochastic to a set of Eulerian fields, which evolve according to stochastic partial differential equations. It has been currently used mostly to non-premixed configurations and in this work we asses its performance for the more challenging case of a flame propagating through different equivalent ratios. The novelty of the work lies in the fact that differential diffusion has been accounted for. One isothermal and one reactive case have been simulated, An overall good agreement is found for the time-averaged velocity and temperature statistics is observed. Although differential diffusion plays a role in the instantaneous flame structure it does not seem to affect the mean quantities as much.

\section{Acknowledgement}

This work was supported by the EPSRC UK Consortium on Turbulent Reacting Flow (UKCTRF) and used the ARCHER UK National Supercomputing Service. (http://www.archer.ac.uk). Dr Vogiatzaki would like to acknowledge the support of the Engineering and Physical Science Research Council through the grant EP/P012744/1. 


\section{References}

[1] Andrei N. Lipatnikov. Stratified turbulent flames: Recent advances in understanding the influence of mixture inhomogeneities on premixed combustion and modeling challenges. Progress in Energy and Combustion Science, 62(Supplement C):87 - 132, 2017.

[2] M. Besson, P. Bruel, J.L. Champion, and Deshaies B. Experimental analysis of combusting flows developing over a plane-symmetric expansion. Journal of Thermophysics and Heat Transfer, 14(1):59-67, 2000.

[3] P Anselmo-Filho, S Hochgreb, R S Barlow, and R S Cant. Experimental measurements of geometric properties of turbulent stratified flames. Proc. Combust. Inst., 32(2):1763-1770, 2009.

[4] R.S. Barlow, M.J. Dunn, M.S. Sweeney, and S. Hochgreb. Effects of preferential transport in turbulent bluff-body-stabilized lean premixed ch4/air flames. Combustion and Flame, 159(8):2563 - 2575, 2012.

[5] M.S. Sweeney, S. Hochgreb, M. J. Dunn, and R.S. Barlow. The structure of turbulent stratified and premixed methane/air flames i: Non-swirling flows. Combustion and Flame, 159(9):2896 - 2911, 2012.

[6] M.S. Sweeney, S. Hochgreb, M.J. Dunn, and R.S. Barlow. The structure of turbulent stratified and premixed methane/air flames ii: Swirling flows. Combustion and Flame, 159(9):2912 - 2929, 2012.

[7] M. S. Sweeney, S. Hochgreb, M. J. Dunn, and R.S. Barlow. Multiply condi- 
tioned analyses of stratification in highly swirling methane/air flames. Combustion and Flame, 160(2):322 - 334, 2013.

[8] R. Zhou, S. Balusamy, M.S. Sweeney, R. S. Barlow, and S. Hochgreb. Flow field measurements of a series of turbulent premixed and stratified methane/air flames. Combustion and Flame, 160(10):2017 - 2028, 2013.

[9] F. Seffrin, F. Fuest, D. Geyer, and A. Dreizler. Flow field studies of a new series of turbulent premixed stratified flames. Combust. Flame, 157(2):384 $-396,2010$.

[10] B. Bhm, J.H. Frank, and A. Dreizler. Temperature and mixing field measurements in stratified lean premixed turbulent flames. Proc. Combust. Inst., 33(1):1583 - 1590, 2011.

[11] B. Renou, E. Samson, and A. Boukhalfa. An experimental study of freely propagating turbulent propane/air flames in stratified inhomogeneous mixtures. Combustion Science and Technology, 176(11):1867-1890, 2004.

[12] N. Pasquier, B. Lecordier, M. Trinit, and A. Cessou. An experimental investigation of flame propagation through a turbulent stratified mixture. Proceedings of the Combustion Institute, 31(1):1567 - 1574, 2007.

[13] P. Anselmo-Filho, S. Hochgreb, R.S. Barlow, and R.S. Cant. Experimental measurements of geometric properties of turbulent stratified flames. Proceedings of the Combustion Institute, 32(2):1763 - 1770, 2009.

[14] D.C. Haworth, R.J. Blint, B. Cuenot, and T.J. Poinsot. Numerical simula- 
tion of turbulent propane-air combustion with nonhomogeneous reactants. Combust. Flame, 121(3):395-417, 2000.

[15] C. Jimnez, B. Cuenot, T. Poinsot, and D. Haworth. Numerical simulation and modeling for lean stratified propane-air flames. Combust. Flame, 128(12):1-21, 2002.

[16] A.Y. Klimenko and R.W. Bilger. Conditional moment closure for turbulent combustion. Progress in Energy and Combustion Science, 25(6):595 - 687, 1999.

[17] A.X. Sengissen, J.F. Van Kampen, R.A. Huls, G.G.M. Stoffels, J.B.W. Kok, and T.J. Poinsot. Les and experimental studies of cold and reacting flow in a swirled partially premixed burner with and without fuel modulation. Combust. Flame, 150(1-2):40-53, 2007.

[18] C. Duwig and C. Fureby. Large eddy simulation of unsteady lean stratified premixed combustion. Combust. Flame, 151(1-2):85 - 103, 2007.

[19] S. Nambully, P. Domingo, V. Moureau, and L. Vervisch. A filtered-laminarflame pdf sub-grid-scale closure for les of premixed turbulent flames: Ii. application to a stratified bluff-body burner. Combustion and Flame, 161(7):1775 - 1791, 2014.

[20] A. Donini, R.J.M. Bastiaans, J.A. van Oijen, and L.P.H. de Goey. Differential diffusion effects inclusion with flamelet generated manifold for the modeling of stratified premixed cooled flames. Proceedings of the Combustion Institute, 35(1):831 - 837, 2015. 
[21] J.C. Massey, I. Langella, and N. Swaminathan. Large eddy simulation of a bluff body stabilised premixed flame using flamelets. Flow, Turbulence and Combustion, 2018.

[22] A. Roux and H. Pitch. Large-eddy simulation of stratified combustion. Annual Research Briefs, Center for Turbulence Research, pages 275-288, 2010.

[23] G. Kuenne, F. Seffrin, F. Fuest, T. Stahler, A. Ketelheun, D. Geyer, Janicka, J., and A. Dreizler. Experimental and numerical analysis of a lean premixed stratified burner using 1D Raman/Rayleigh scattering and large eddy simulation. Combust. Flame, 159():26692689, 2012.

[24] F. Cavallo Marincola, T. Ma, and A.M Kempf. Large eddy simulations of the darmstadt turbulent stratified flame series. Proc. Combust. Inst, 34:13071315, 2013.

[25] P. Trisjono, K. Kleinheinz, S. Kang, and H. Pitsch. Large eddy simulation of stratified and sheared flames of a premixed turbulent stratified flame burner using a flamelet model with heat loss. Flow Turbulence Combust, 92:201235, 2014.

[26] S.B. Pope. PDF methods for turbulent reactive flows. Prog. Energy Combust. Sci., 11(2):119-192, 1985.

[27] T Brauner, W P Jones, and A J Marquis. LES of Turbulent Premixed and Stratified Combustion using a Sub-Grid PDF Method. Submitted, 2018. 
[28] J. Smagorinsky. General circulation experiments with the primitive equations. Journal of Monthly Weather Review, 91:99-164, 1963.

[29] U. Piomelli and Junhui Liu. Large-eddy simulation of rotating channel flows using a localized dynamic model. Physics of Fluids, 7(4):839 - 48, 1995.

[30] J. O. Hirschfelder and C. F. Curtiss. Proc. Combust. Inst., 1949.

[31] T. P. Coffee and J. M. Heimerl. Transport algorithms for premixed, laminar steady-state flames. Combustion and Flame, 43(1):273-289, 1981.

[32] F. Gao and E.E. O'Brien. A large eddy simulation scheme for turbulent reacting flows. Physics of Fluids A, 5:1282-1284, 1993.

[33] T Brauner, W P Jones, and A J Marquis. LES of the Cambridge Stratified Swirl Burner using a Sub-grid pdf Approach. Flow Turbulence Combust, 96(4):965-985, 2016.

[34] C. Dopazo and E. O'Brien. Functional formulation of nonisothermal turbulent reactive flows. Phys. Fluids, 17(1968), 1974.

[35] W. P. Jones and S. Navarro-Martinez. Large eddy simulation of autoignition with a subgrid probability density function method. Combustion and Flame, 150(3):170-187, 82007.

[36] M.A. Picciani, E.S. Richardson, and S. Navarro-Martinez. Resolution requirements in stochastic field simulation of turbulent premixed flames. Flow, Turbulence and Combustion, 2018. 
[37] W. P. Jones and V.N. Prasad. Large eddy simulation of the sandia flame series (d-f) using the eulerian stochastic field method. Combust. Flame, 157(9):1621 - 1636, 2010. 

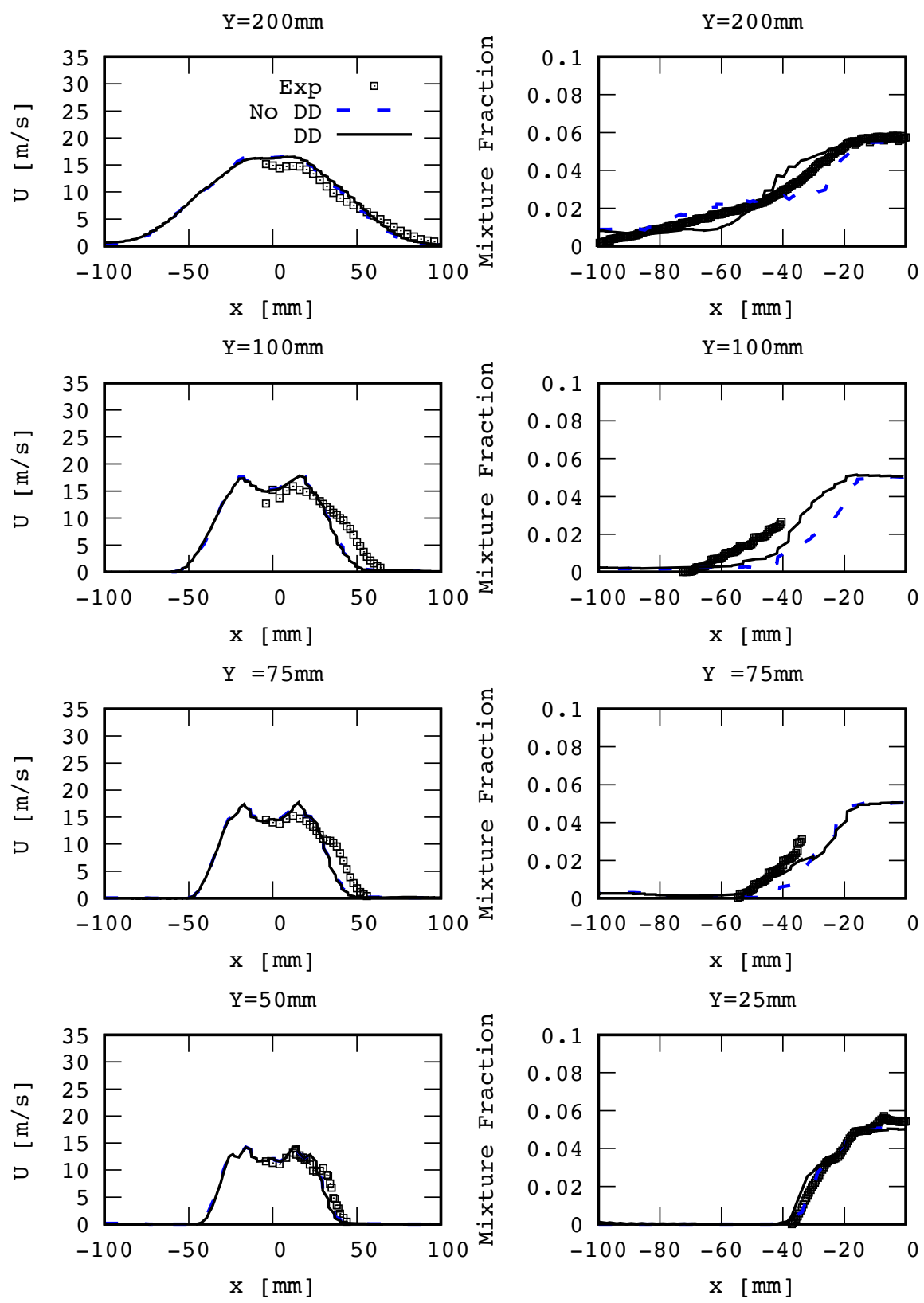

Figure 7: Radial profiles of axial velocity and mixture fraction at four axial location for $T S F A_{r}$. Squares represent experimental data, solid lines simulations with differential diffusion and dashed line simulations without differential diffusion 

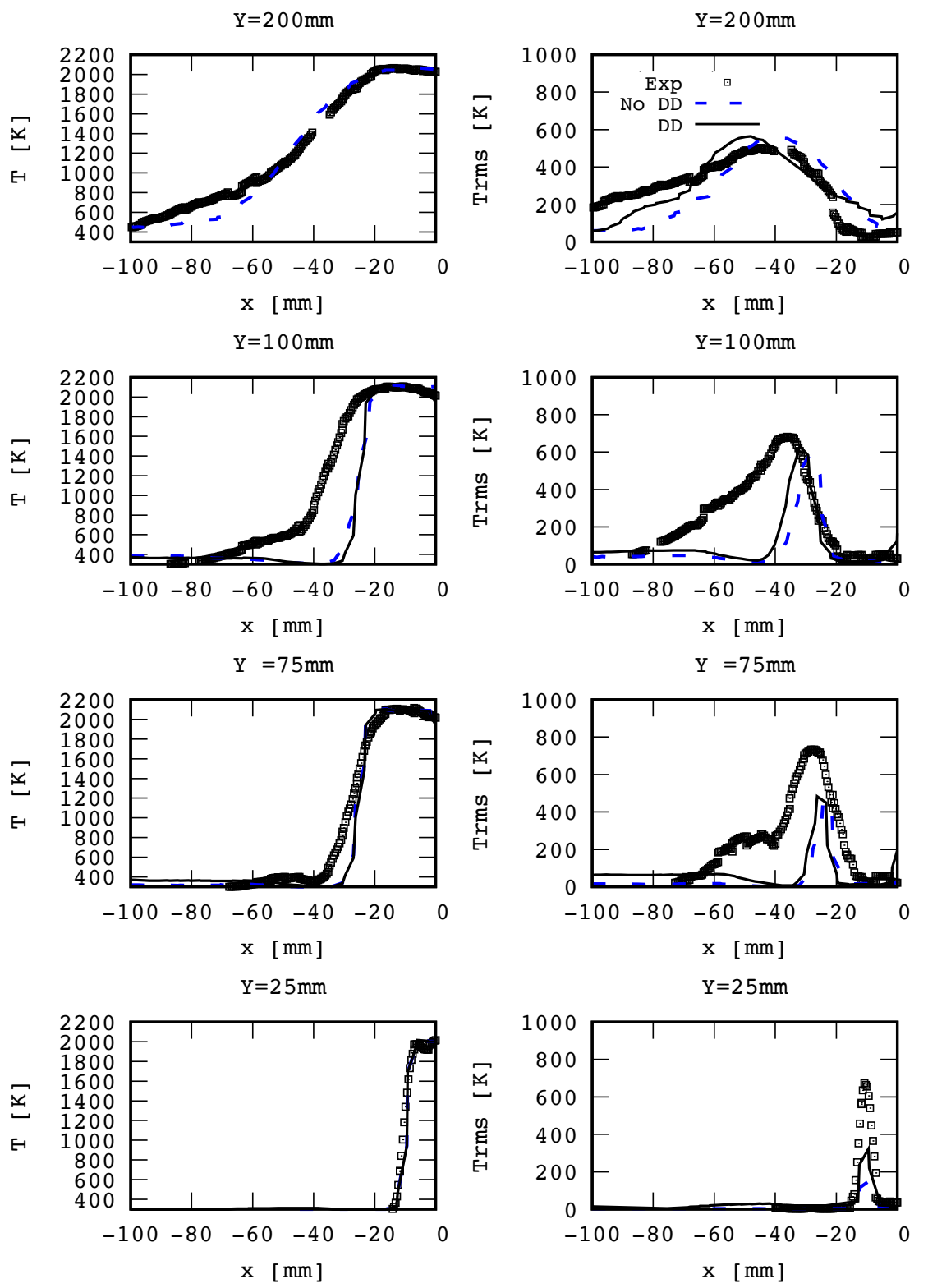

Figure 8: Radial profiles of temperature and temperature rms at four axial location for $T S F A_{r}$. Squares represent experimental data, solid lines simulations with differential diffusion and dashed line simulations without differential diffusion 